\title{
Diarios locales: muchos cambios y poco periodismo
}

\section{Xosé López}

Profesor de Periodismo.

Universidad de Santiago de Compostela

\section{Resumen:}

Los diarios locales encuentran cada vez más problemas para incrementar el número de compradores e, incluso, para mantenerlos y evitar pérdidas importantes. Después de una década de cambios en el diseño y en los contenidos, los resultados de los estudios realizados indican que los nuevos lectores llegan con "muchas dificultades, a cuenta gotas", mientras pierden suscriptores, en muchos casos por razones biológicas. La competencia de los gratuitos y de las ediciones digitales en la red multiplican los problemas de un sector que aumenta el número de cabeceras pero que no es capaz de dar respuestas satisfactorias a muchos de los retos que tiene planteados. A pesar de los muchos cambios intentados en los últimos años, no han potenciado suficientemente el trabajo periodístico. Algunos creen que deben volver a un "periodismo puro y duro" muy cercano que ofrezca informaciones útiles a los lectores y que combine la edición en papel con propuestas digitales en la red y con medios gratuitos dirigidos a segmentos muy definidos del mercado.

Palabras clave:

Prensa local, periodismo, medios de comunicación impresos.

\section{Abstract:}

Local newspapers have nowadays many problems to increase their number of readers, and even to keep them and avoid severe losses. After a decade of changes in design and content, new readers hardly arrive, while newspapers lose suscriptors, for biological reasons in many cases. Competence of free and online press multiply the problems of this sector, which increases the number of newspapers but is incapable to give satisfactory answers to its current challenges. Although the changes introduced in the last decade, local press did not potenciated enough the journalist work. Some believe that they should return to a 
'traditional journalism', very close to its readers, to whom provide useful information. It should also combine written publishing with online proposals, and free media directed towards defined segments of the market.

Key words:

Local press, Journalism, Press media.

\section{Introducción}

Los estudios sobre los medios impresos a lo largo de la historia han puesto de manifiesto la dimensión local como un factor básico y permanente del periodismo. Muy pocos rotativos en el mundo no son, en alguna medida, locales. Y, en el Estado español, los diarios locales figuran como un elemento histórico y genuino del periodismo (Guillamet, 2002: 183). Siempre han prestado especial atención a la información cercana y, de acuerdo con sus principios editoriales, han tratado de responder a la información que el ciudadano necesita. No siempre lo han conseguido. El análisis de su discurso, entendido en el sentido fijado por Teun A. Van Dijk (2000: 245) -el análisis simultáneo de la semántica del texto y de las estrategias discursivas de los actores, teniendo presente que esta articulación permite vincular la representación textual de la realidad al proceso social y político de su producción-, nos ha mostrado múltiples distorsiones, omisiones o representaciones sociales de un grupo, es decir, sesgadas.

En este comienzo de siglo, cuando las transformaciones en el hipersector de la comunicación revisten especial importancia, el diario local corre el riesgo de no evolucionar con los tiempos, con la sociedad a la que informa y a la que debe ofrecer información útil para que los ciudadanos puedan atender las necesidades que tienen en la vida cotidiana. Los rasgos definidores de la prensa local española de los últimos diez años le han dado una personalidad con la que ahora tiene que afrontar los nuevos desafíos, entre los que destaca la recuperación del periodismo "puro y duro", la capacidad de innovación y una mejor comunicación con los destinatarios. Esos productos, así como el periodismo que se hace en ellos, han construido su identidad a partir de patrones originarios de Estados Unidos y Europa (Marques de Melo/Queiroz, 1998: 11), a los que han añadido un fuerte "sello" construido a partir de la herencia del periodismo español del último siglo.

La prensa local, con su identidad en cada ámbito territorial, ha mantenido una evolución en estos diez años que ha mostrado un crecimiento en los mercados, cambios textuales y formales, y algunos pasos de las estrategias que ha seguido para cumplir con su papel en la sociedad, donde ha intentado cosechar rentabilidad económica y social. A fin de conocer esos cambios, hemos analizado la evolución de la difusión en cada zona y la interrelación existente entre esos diarios y los procesos de vertebración territorial, cultural y política. Para ello hemos tenido como punto de partida las investigaciones realizadas en el País Vasco y en Cataluña sobre la prensa en los últimos diez años, especialmente los coordinados 
por Mikel Arriaga y Salvador Cardús, respectivamente, y los informes sobre la evolución de los medios impresos, principalmente los dirigidos por Bernardo Díaz Nosty en tres años (1995, 2001 y 2004). Los datos sobre análisis textual y formal, preparados a partir del diseño metodológico realizado por José Ignacio Armentia y su equipo (2000) -una posterior investigación publicada en 2002, y un artículo de Armentia y Caminos publicado en 2003, desarrolla aspectos relativos al diario de servicios-, los hemos realizado sobre los diarios de Galicia y sobre una muestra de veinte cabeceras locales del Estado, en un proyecto financiado por el Plan Gallego de Investigación y Desarrollo Tecnológico de la Xunta de Galicia.

\section{La confirmación del liderazgo}

El análisis de la difusión de la prensa diaria en el Estado español desde 1995 a 2005 confirma la buena evolución de las cabeceras locales en sus respectivos mercados, donde ya venían manteniendo un crecimiento significativo desde 1976. En el año 1999, a las puertas del nuevo milenio, en treinta y ocho provincias el liderazgo de difusión recae en cabeceras locales o regionales, mientras en veintiuna demarcaciones corresponde la segunda posición a títulos de la misma definición. Diecinueve diarios regionales y locales, líderes en sus ámbitos de difusión, consiguen acaparar más del 50 por ciento del mercado de la información general en sus respectivas demarcaciones (Díaz Nosty, 2001: 111). Ideal de Granada, en Granada; El Diario Vasco, en Guipúzcoa; Hoy, en Badajoz; La Voz de Galicia, en A Coruña; Diario de Navarra, en Pamplona; El Norte de Castilla, en Valladolid, y Heraldo de Aragón, en Zaragoza, tenían más del 65 por ciento del mercado de las citadas demarcaciones.

Los datos a finales de 2004 apenas habían variado. A pesar del escaso número de lectores jóvenes que se ha incorporado a la lista de compradores de diarios, lo cierto es que la difusión de los periódicos se ha incrementado en el año 2003 y en 2004, con un destacado aumento, según los primeros avances de datos provisionales de difusión de 2004. La explicación de este comportamiento de las cabeceras locales en los mercados no ofrece dudas. Fueron las promociones las que evitaron que la tendencia mundial al descenso de las ventas, fijado en el informe mundial de la WAN correspondiente a 2004 en un 0,1 por ciento (WAN, 2004: 2-5), afectase a estas cabeceras y tuviese un impacto negativo en los medios del Estado español. Es decir, el mercado de la prensa de pago en el área de la información general parece que ha tocado techo y que difícilmente crecerá en el futuro.

Estos indicadores de la difusión no impiden, sin embargo, que se incremente el número de cabeceras. En los últimos años han aparecido diarios promovidos por empresarios locales, como El Pueblo de Albacete (2003), La Prensa de Zamora (2003), Noticias de La Rioja (2004)..., o por grupos de comunicación con mayor o menor tamaño, como El Día de Ciudad Real (2002), Heraldo de Castellón (2003), La Opinión de Granada (2003), El Correo de Málaga (2003) -desaparecido en 2005-, Granada Hoy (2003), La Voz de Cádiz (2004), Diario de Noticias de Álava (2004)... El número de nuevos diarios ha sido especialmente significativo en el año 2004, reflejo de la disputa entre los principales grupos por alguno de 
los mercados locales, sobre todo de Andalucía. En cambio, en el apartado de desapariciones relevantes en el mapa mediático tenemos pocas: El Periódico de Gijón (2003) y La Prensa de Zamora (2004); y en el de sustituciones, la de Berria por el clausurado Euskaldunon Egunkaria (2003).

\begin{tabular}{|l|c|c|}
\hline \multicolumn{1}{|c|}{ Cabeceras aparecidas en 2004 } & Ciudad & Empresa editora \\
\hline Diario de Noticias de Álava & Álava & Izoria Ediciones 2004 \\
\hline La Estafeta de Navarra (desapareció en 2005) & Pamplona & $\begin{array}{c}\text { Editora Independiente } \\
\text { de Medios de Navarrra }\end{array}$ \\
\hline La Voz de Cádiz & Cádiz & Grupo Vocento \\
\hline Málaga Hoy & Málaga & Grupo Joly \\
\hline Noticias de La Rioja & Logroño & Promersa \\
\hline
\end{tabular}

Fuente: Elaboración propia.

\section{Cambios en la presentación}

Los diarios locales, en esta fase de consolidación del liderazgo en el mercado, resistiendo el envite de la competencia tradicional y, en algunos casos, a las nuevas cabeceras, han experimentado importantes cambios en la presentación, profundizando en una tendencia que han seguido en los últimos diez años: el modelo de diario de servicios. A partir de la década de los ochenta del pasado siglo, en las sociedades occidentales del bienestar, los diarios prestan menos atención a las grandes cuestiones socio-económicas y políticas y, en cambio, dedican más espacio a las cuestiones cotidianas de los lectores: la salud, el medio ambiente, la alimentación, los viajes... (Armentia/Caminos, 2003: 12). Y, desde el punto de vista del diseño, realizan cambios para un lector medio con poco tiempo para examinar la prensa y que busca una información más gráfica, más visual.

Los principales diarios locales y regionales realizaron cambios en el diseño entre los años 2000 y 2004. En los veinte diarios seleccionados ${ }^{1}$ para la muestra del "estudio en profundidad" de la investigación que sirve de base a este artículo hubo renovación en el diseño para mejorar la información de

\footnotetext{
${ }^{1}$ La investigación de los diarios locales tiene dos partes. En una primera se analizaron todos los diarios de Galicia, mientras en una segunda se estudiaron diecisiete de los principales diarios del Estado controlados por la división de OJD (Oficina de Justificación de la Difusión) de Información y Control de Publicaciones y otros tres sin control de la difusión por OJD. La relación de los diarios controlados por OJD la integran El Correo Español/EPV, La Voz de Galicia, Diario de Navarra, La Nueva España, Las Provincias, Faro de Vigo, La Verdad, El Norte de Castilla, Sur, Ideal de Granada, Diario de Cádiz, Diario de Sevilla, La Rioja, Diario de León, Regió 7, Diari de Girona y Diario de Ávila. Los diarios sin control de OJD analizados son El Correo Gallego, Málaga Hoy y La Voz de Cádiz.
} 
servicios y para hacer más atractiva la imagen visual del producto impreso. La mayoría de las cabeceras estudiadas estrenó rediseño en los años 2003 y 2004, al tiempo que mantuvieron proyectos para emprender pequeñas actualizaciones dirigidas a reforzar la personalidad de los productos y a responder a las nuevas demandas de los lectores.

Año del cambio de diseño de los diarios analizados

\begin{tabular}{|l|c|c|}
\hline \multicolumn{1}{|c|}{ Cabeceras } & Localidad & $\begin{array}{c}\text { Año del último } \\
\text { cambio de diseño }\end{array}$ \\
\hline El Correo Español/EPV & Bilbao & 2000 \\
\hline La Voz de Galicia & A Coruña & 2002 \\
\hline Diario de Navarra & Pamplona & 2003 \\
\hline La Nueva España & Oviedo & 2004 \\
\hline Las Provincias & Valencia & 2001 \\
\hline La Verdad & Murcia & 2003 \\
\hline El Norte de Castilla & Valladolid & 2000 \\
\hline Sur & Málaga & 2002 \\
\hline Ideal Granada & Granada & 2001 \\
\hline Diario de Cádiz & Cádiz & 2003 \\
\hline Diario de Sevilla & Sevilla & 2003 \\
\hline La Rioja & Logroño & 2003 \\
\hline Diario de León & León & 2003 \\
\hline Regió 7 & Manresa & 2004 \\
\hline Diari de Girona & Girona & 2004 \\
\hline Diario de Ávila & Ávila & 2003 \\
\hline El Correo Gallego & Santiago de Compostela & 2002 \\
\hline Málaga Hoy & Málaga & 2004 \\
\hline La Voz de Cádiz & Cádiz & 2004 \\
\hline Faro de Vigo & & \\
\hline Fuo & \\
\hline
\end{tabular}

Fuente: Elaboración propia. 
De acuerdo con las conclusiones de la investigación, basada en el análisis de los productos y en las entrevistas realizadas a los responsables de la redacción de los diarios locales y regionales, hay una coincidencia general sobre el diario que quieren elaborar: "Hagamos un periódico atractivo visualmente, con la profundidad que no da la televisión, y contemos las historias que más les afectan a los ciudadanos. Prestemos menos atención a los políticos y más a la sociedad civil. Retratemos la ciudad, comarca o región en la que vivimos de forma que nuestros compradores se sientan reflejados en las páginas del diario". Es toda una declaración de intenciones que se repite, pero que, como todo deseo, la práctica se encarga de sumarle matices y, muchas veces, de contradecirlo.

Ciertamente, la información es "más transparente", los cambios se han orientado a una mayor jerarquización interna y a una mayor descomposición en paquetes principales y secundarios para facilitar una lectura rápida y diferenciar información y documentación. Pero a veces los grandes temas del día tienen poca profundidad o se introducen elementos visuales que generan "efectos mosaico" que pueden despistar al lector. Hay, pues, muchos aspectos que todavía deberán mejorar en las próximas actualizaciones de diseño.

\section{El valor de lo próximo}

A lo que casi siempre presta atención la prensa local es a la información cercana. Los diarios están convencidos de que el espacio local no es un ámbito minoritario o marginal, sino un sector fundamental en las estrategias de la comunicación moderna (Moragas, 2003: 163). Sobre todo, saben que es su espacio básico de actuación. Quizás por ello el análisis de contenido de los diarios en los últimos diez años confirma la importancia cuantitativa y cualitativa que todas las cabeceras conceden a lo próximo.

El eslogan de un grupo gallego de prensa local, La Capital, resume a la perfección la vocación de la prensa de esta Comunidad y de los principales rotativos analizados: "Las cosas de aquí, contadas desde aquí”. Las secciones de información local ocupan entre un 20 y un 30 por ciento de los contenidos, al tiempo que muchas veces abren el periódico, lo que denota la prioridad que le otorgan. Además, los contenidos de información próxima de todo el producto -se incluye la de economía, deportes, servicios...- se sitúan entre el 60 y el 70 por cien y el 80 y el 90 por cien. Algunas cabeceras sólo tienen entre cinco y diez páginas de contenidos no locales/regionales -es el caso de El Correo Gallego, diario que se entrega inseparablemente con El Mundo en Galicia-.

También hay una tendencia al incremento de la interpretación y de la opinión propia en bastantes cabeceras. El reportaje y la crónica han recuperado terreno, así como la entrevista y los artículos de opinión elaborados por redactores y colaboradores de cada uno de esos medios -casos muy significativos son La Voz de Galicia, El Correo Gallego, Regió 7, Diario de Sevilla, Málaga Hoy...-. La mayoría de las cabeceras cada día elige un tema central al que dedica un tratamiento más profundo, con el empleo de 
varias modalidades expresivas y con opinión. De esta forma, algunos rotativos buscan definir un tema de interés para la deliberación pública.

Lo que no consigue la mayoría de los diarios es desprenderse de esa tendencia hacia la "oficialización" que muchas veces hemos encontrado en el tratamiento de los temas próximos. La representación de las acciones de los portavoces políticos es una de las razones de su amplia cobertura por los medios. Pero, en ocasiones, su presencia en la prensa se debe más a su capacidad organizativa como fuentes que a la relevancia de sus acciones. Con todo, se advierte una mayor presencia de fuentes de la sociedad civil organizada e intentos de buscar una mayor pluralidad. Es una forma de romper la dependencia de las fuentes oficiales, que todavía tienen una fuerte presencia -más de la mitad de las informaciones están elaboradas a partir de fuentes oficiales o con una presencia significativa-.

Como temas emergentes, en el estudio de los contenidos hemos encontrado que el medio ambiente y la sanidad aparecen con más fuerza, así como las tecnologías actuales. La salud ambiental y la humana protagonizan cada vez más informaciones. En cambio, los temas estrictamente políticos pierden espacio, aunque sigue formando parte de las cuestiones básicas para todos los medios locales -hacen un seguimiento con periodistas especializados de la política autonómica y municipal-.

\section{La calidad es cara}

En este escenario de cambios formales, con mucha atención a la presentación, y de cambios textuales -tanto de temas como de modalidades expresivas-, los responsables de los medios de comunicación dicen que han aprendido una lección: la calidad periodística es cada vez más cara. Es por ello que siguen buscando fórmulas para hacer frente a los desafíos y dinero para poder financiar esas nuevas acciones. Sostienen que muchas de las iniciativas que han introducido los diarios en los últimos años han ofrecido resultados positivos, pero no siempre han conseguido soluciones plenamente satisfactorias. Y citan como ejemplo la introducción de jóvenes lectores, que es uno de los objetivos de las principales cabeceras locales, temerosas de que la desaparición de una generación de sus lectores les arrastre a una pérdida de difusión.

Mientras los responsables de comercialización concentran esfuerzos en nuevas promociones, que permiten mantener e incluso a veces incrementar la difusión, los responsables de la redacción miran, aunque sólo algunas veces, hacia las fortalezas del periodismo puro y duro, el de "los buenos tiempos de antaño". Su posición la comparten muchos profesionales, que, sin embargo, sostienen que las empresas no tienen voluntad de invertir en las redacciones para crear equipos capaces de elaborar periodismo de calidad en la compleja sociedad actual. Sus afirmaciones coinciden con un dato: en los últimos cinco años (2000-2004) muy pocos diarios locales han incrementado significativamente sus equipos de redacción. 
En la actual década, muchos diarios han mostrado síntomas de las heridas que aquejan al periodismo y no han sido capaces de ponerles remedio. Aumentan las incorrecciones, las noticias falsas, las informaciones con pocas fuentes... Algunos medios incluso se han instalado en la prepotencia y la arrogancia. Muchas veces, el periodismo, en lugar de tratarse de un servicio a la comunidad, es un aprovechamiento en beneficio propio, de algunos periodistas, pero, sobre todo, y es lo grave, de muchos editores o propietarios de medios (De Pablos, 2001: 8). Es un divorcio que no tiene fácil solución si no hay un cambio radical de rumbo por parte de los diarios dirigido a recomponer su alianza con los ciudadanos y con la comunidad a la que informan y de la que informan.

Aquellos diarios que quieran hacer periodismo de calidad en el futuro tienen, por tanto, que introducir ese giro en su estrategia económica a fin de volver a los viejos principios y recuperar los objetivos de servicio a la comunidad. Y, como segundo paso, deberán destinar más recursos a la redacción, porque el periodismo de calidad es caro. El discurso del poder crea ilusiones falsas que persiguen la formación paulatina del ciudadano consumidor (Reig, 2004: 3006) y los medios no pueden transmitir sólo ese mensaje. Los ciudadanos tienen necesidades, derechos e intereses legítimos y, además, son los sujetos de la información.

Ciertamente, los medios impresos tienen más competencia en el mercado, lo que les crea dificultades añadidas en el campo económico. La masiva llegada de gratuitos a los escenarios locales y las ediciones digitales les obliga a la redefinición de estrategias tanto como empresas de comunicación, con programas muy definidos de diversificación, como al análisis de los contenidos que difunden y los que pueden incorporar en el futuro. Es, pues, un problema que afecta a la estrategia económica y la estrategia periodística.

\section{A modo de conclusión}

El análisis formal y textual de los diarios locales nos permite asegurar que no corren buenos tiempos para el periodismo. El negocio es lo primero en un mercado local cada vez más disputado. Hay que vender, aunque sea basura, aunque sea mentira (Diezhandino, 2003: 94). En estos últimos diez años los diarios han realizado importantes cambios tanto en la presentación como en los contenidos, pero mantienen muchos de los problemas que les han aquejado durante buena parte de la segunda mitad del siglo XX. Los productos han mejorado notablemente, como reconocen los responsables de los medios -el 100 por cien-, los profesionales de la información -el 70 por cien-y los lectores -el 85 por cien-. Pero no han encontrado un modelo que devuelva el periodismo a un lugar central al servicio de la sociedad.

Del estudio de los principales diarios locales y regionales, deducimos que, a pesar de los muchos cambios que intentó la mayoría de las cabeceras en esta década, sus proyectos no han conseguido la potenciación efectiva del trabajo periodístico. Quizás por ello profesionales y lectores creen que ha lle- 
gado la hora de volver a un "periodismo puro y duro" muy cercano que ofrezca informaciones útiles a los lectores y que combine la edición en papel con propuestas digitales en la red y con medios gratuitos dirigidos a segmentos muy definidos del mercado.

Lo que no cabe duda es que existe un distanciamiento entre muchos lectores y los medios locales que puede terminar en ruptura. De momento, los diarios consiguen, mediante promociones y la actualización de los productos, pequeños pasos adelante con los que responden a problemas puntuales. Pero, a juzgar por sus valoraciones sobre el estado de la cuestión, son conscientes de que el ámbito local es un espacio estratégico en la Sociedad de la Información y el Conocimiento, pero también admiten que sólo tendrán éxito si son capaces de atender las nuevas demandas ciudadanas. Y en ese punto apenas han actuado de momento porque han sido incapaces de definir el nuevo papel de los diarios locales y las características del nuevo modelo de producto.

Sin olvidar la importancia de seguir renovando las cuestiones textuales y formales, los principales diarios locales vuelven los ojos hacia el periodismo "puro y duro". Saben que ahí pueden encontrar alguna receta para introducir un giro radical a lo que ha sido una constante en la última década: muchos cambios y poco periodismo.

\section{Referencias bibliográficas}

- Arriaga, M. y Pérez Soengas, J. L. (2000): La prensa diaria en Euskal Herria (1976-1998), Bilbao: Universidad del País Vasco.

- Armentia, J. I. et al. (2000): El diario digital. Análisis de los contenidos textuales, aspectos formales y publicitarios, Barcelona: Bosch.

- Armentia, J. I. y Caminos, J. M. (2003): “Los formatos de lectura rápida como seña de identidad visual del diario de servicios”, doxa comunicación, n. ${ }^{\circ}$ 1, Madrid, pp. 12-33.

- Caminos, J. M. et al. (2002): El diario de servicios en España, Oviedo: Septem.

- Cardús, S. (1995): Premsa i poder a Catalunya 1981-1992, Barcelona: Edicions La Campana.

- Moragas, M. de (2003): "Do global ó local como referente mediático. A aposta polos gratuítos", pp. 161-179, en VV. AA: Medios locais e prensa gratuita, Santiago de Compostela: Xunta de Galicia.

- De Pablos Coello, J. M. (2001): El periodismo herido, Madrid: Foca.

- Diaz Nosty, B. (2004): Informe anual de la profesión periodística, Madrid: Asociación de la Prensa de Madrid.

- (2001): Informe anual de la Comunicación 2000-2001. Estado y tendencias de los medios en España, Barcelona: Grupo Zeta.

- (1995): Comunicación Social/ Tendencias 1995, Madrid: Fundesco. 
- Diezhandino, P. (2003): "Periodismo y nueva economía. La historia de un fracaso", doxa comunicación, n. ${ }^{\circ} 1$, Madrid, pp. 81-94.

- Guillamet, J. (2002): “Pasado y futuro de la prensa local”, pp. 181-196, en López Lita, R., Fernández Beltrán, F. y Durán, A. (eds.): La prensa local y la prensa gratuita, Castellón de la Plana: Publicaciones de la Universidad Jaume I.

- Marques de Melo, J. y Queiroz, A. (1998): Identidade da impresa brasileira no final de século. Das estratégias comunicacionais aos enraizamentos e ás ancoragens culturais, Sâo Paulo: UNESCO/Umesp de Comunicaçâo.

- Reig, R. (2004): Dioses y diablos mediáticos. Cómo manipula el poder a través de los medios de comunicación, Barcelona: Ediciones Urano.

- Van Dijk, T. A. (2000): Ideología. Una aproximación multidisciplinaria, Barcelona: Gedisa.

- WAN (2004): World Press Trends 2004, París: World Association of Newspapers (WAN). 